\title{
Feasibility study on mouse live imaging after spinal cord injury and poly(lactide-co-glycolide) bridge implantation
}

\author{
Andrea Anzalone \\ Jenu V. Chacko \\ Rebecca A. Nishi \\ Courtney Dumont \\ Dominique Smith \\ Lonnie D. Shea \\ Michelle A. Digman \\ Brian J. Cummings \\ Aileen J. Anderson
}




\title{
Feasibility study on mouse live imaging after spinal cord injury and poly(lactide-co-glycolide) bridge implantation
}

\author{
Andrea Anzalone, ${ }^{a, \dagger}$ Jenu V. Chacko, ${ }^{b, t, \ddagger}$ Rebecca A. Nishi, ${ }^{a, c}$ Courtney Dumont, ${ }^{\text {d,e }}$ Dominique Smith, ${ }^{d, e}$ \\ Lonnie D. Shea, ${ }^{d, e}$ Michelle A. Digman, ${ }^{b, f}$ Brian J. Cummings, ${ }^{a, c, g, h, \dagger}$ and Aileen J. Anderson ${ }^{a, c, g, h, t, *}$ \\ aUniversity of California, Institute for Memory Impairments and Neurological Disorders, Irvine, California, United States \\ bUniversity of California Irvine, Department of Biomedical Engineering, Irvine, California, United States \\ 'University of California, Sue and Bill Gross Stem Cell Research Center, Irvine, California, United States \\ dUniversity of Michigan, Department of Chemical Engineering, Ann Arbor, Michigan, United States \\ eUniversity of Michigan, Department of Biomedical Engineering, Ann Arbor, Michigan, United States \\ fUniversity of California Irvine, Laboratory for Fluorescence Dynamics, Irvine, California, United States \\ gUniversity of California, Department of Physical Medicine and Rehabilitation, Irvine, California, United States \\ hUniversity of California, Department of Anatomy and Neurobiology, Irvine, California, United States
}

\begin{abstract}
Spinal cord injury $(\mathrm{SCl})$ causes permanent paralysis below the damaged area. $\mathrm{SCl}$ is linked to neuronal death, demyelination, and limited ability of neuronal fibers to regenerate. Regeneration capacity is limited by the presence of many inhibitory factors in the spinal cord environment. The use of poly(lactide-co-glycolide) (PLG) bridges has demonstrated the ability to sustain long-term regeneration after $\mathrm{SCl}$ in a cervical hemisection mouse model. Critically, imaging of regenerating fibers and the myelination status of these neuronal filaments is a severe limitation to progress in $\mathrm{SCl}$ research. We used a transgenic mouse model that selectively expresses fluorescent reporters (eGFP) in the neuronal fibers of the spinal cord. We implanted a PLG bridge at C5 vertebra after hemisection and evaluated in live animals' neuronal fibers at the bridge interface and within the bridge 8 weeks postimplant. These in vivo observations were correlated with in situ evaluation 12 weeks postimplantation. We sectioned the spinal cords and performed fluorescent bioimaging on the sections to observe neuronal fibers going through the bridge. In parallel, to visualize myelination of regenerated axons, we exploited the characteristics of the third-harmonic generation arising from the myelin structure in these fixed sections. @2018 Society of Photo-Optical Instrumentation Engineers (SPIE) [DOI: 10.1117/1.JBO.23.6.065007]
\end{abstract}

Keywords: spinal cord injury; live imaging; fluorescence; regeneration; biomaterials.

Paper 180067RR received Jan. 29, 2018; accepted for publication Jun. 11, 2018; published online Jun. 29, 2018.

\section{Introduction}

Spinal cord injury (SCI) is a severe medical condition resulting in motor and sensory deficits, with dramatic functional and socioeconomic impacts. ${ }^{1}$ The pathophysiology of SCI includes axonal severing and loss of neuronal fibers, with failure to sustain a regenerative response that can reconnect long descending motor tracts and ascending sensory fibers. Multiple longterm factors contribute to regeneration failure. After injury, the axons originating in descending cortical and subcortical tracts exhibit dieback over one or more segments, but do not degenerate to the cell body. Additionally, glial scar formation results in a chemical and physical barrier to reconnection of descending motor axons below the lesion..$^{2-4}$ We have recently demonstrated the capacity of poly(lactide-co-glycolide) (PLG) bridges implanted into a $\mathrm{C} 5$ cervical hemisection injury to promote regeneration of the descending corticospinal tract (CST). In that study, CST fibers expressing a green fluorescent protein (GFP) reporter exhibited growth through the bridge and into the distal spinal cord, ${ }^{5}$ supporting implantation of PLG bridges as

\footnotetext{
*Address all correspondence to: Aileen J. Anderson, E-mail: aja@uci.edu

tThese authors contributed equally.

${ }^{\ddagger}$ Current address: University of Wisconsin, Laboratory for Optical and Computational Instrumentation, Madison, Wisconsin, United States.
}

a substrate for axonal regeneration. ${ }^{6}$ Implantation of this type of scaffolding biomaterial is applicable to SCI models, in which a surgical incision has been made to remove a segment or more of spinal cord, and potentially to traumatic clinical injuries, in which there has been penetration of the gray and white matter of the spinal cord. Current clinical applications of PLG include advanced drug delivery systems, dental sciences, and biodegradable bone grafts. ${ }^{7}$

Evaluation of axonal damage, myelination, and axonal regeneration is critical to investigate regeneration dynamics after SCI or in other implantation paradigms. Accordingly, we sought to develop a tool to identify neuronal fiber growth in a mouse model of SCI, using confocal and two-photon imaging on live animals. This imaging approach demonstrates proof of concept for in vivo imaging within the spinal cord lesion and regenerative fibers with the potential to evaluate remyelination on in situ spinal cord section. In vivo imaging studies of axon trajectories and neuronal fibers after SCI enable unambiguous identification of spared, injured, and regenerating neuronal fibers, providing a strong stage to assess therapies aimed at spurring neuronal fiber growth. ${ }^{8}$ We used Thy1-enhanced green fluorescent protein (eGFP) mice, which have previously been employed in other neurobiological studies and are commercially 
available. ${ }^{9,10}$ These mice express eGFP in neuronal areas, including the spinal cord, enabling visualization of axons of multiple origins. We used a modified upright two-photon microscope equipped for live animal imaging, capable of visualizing fluorescent emission originating from eGFP. We used the same microscope to visualize third-harmonic emission signals arising from myelin on spinal cord sections. ${ }^{11,12}$ Myelin is a compact fatty structure that wraps axons to enhance the speed of signal transmission. ${ }^{13}$ Myelin can be detected via third-harmonic emission signals in the intact spinal cord; in contrast, myelin identifying dyes can only be applied in fixed tissue sections and disrupt its lipid structure. ${ }^{12}$ We visualized fluorescence inside the intact spinal cord rostral and caudal to the lesion site, as well as that inside the PLG bridge arising from regrown neuronal fibers. We then sectioned the spinal cord and visualized the eGFP fluorescence signal through the PLG bridge, and we evaluated the third-harmonic signal to investigate possible fibers remyelination. Third-harmonic generation (THG) is a suitable method to detect myelin inside the central nervous system (CNS). ${ }^{12,14}$ THG can be detected from myelin in a label-free imaging modality that provides micrometer resolution imaging of myelin in the mouse spinal cord. ${ }^{14,12}$ Using this method, we tried to assess axonal myelination adjacent to and inside the PLG bridge. We were able to visualize third-harmonic signal rostral to the bridge entrance, but not inside the bridge. However, our qualitative results show it is possible using reporter mice to visualize a time-lapse regrowth of spinal cord fibers through a PLG bridge.

\section{Methods}

\subsection{Poly(Lactide-Co-glycolide) Bridge}

Synthetic PLG bridges were fabricated by adapting a previously established gas foaming/particulate leaching procedure to include caramelized sucrose fibers as a channel template. ${ }^{6,15,16}$ The final bridge dimensions were $1.25 \mathrm{~mm}$ in height, $1.15 \mathrm{~mm}$ in length (R-C), and $0.75 \mathrm{~mm}$ in width (M-L) [Fig. 2(a)]. The channels were characterized and bridge diameters measured $(n=6)$ using light microscopy images (Leica Microsystems, Wetzlar, Germany) and ImageJ software. The cross-sectional area occupied by the channels and the porosities of these bridges were calculated using equations established previously. ${ }^{17}$ Bridges were imaged using the field emission scanning electron microscopy (Zeiss Leo Gemini 1525, Jena, Germany) at $10 \mathrm{kV}$ after coating with osmium tetra oxide.

\subsection{Spinal Cord Injury and Animals}

All animal housing conditions, surgical procedures, and postoperative care were conducted according to IACUC guidelines at the University of California, Irvine. Female mice were anesthetized using isoflurane (2\%). Mice received a C5 laminectomy, followed by a left-sided lateral hemisection and removal of a unilateral 1- to 1.1-mm segment of the spinal cord to enable bridge implantation into the resulting gap [Fig. 2(c)]. In some instances, the gap was too wide in the medial-lateral direction, and two bridges were placed into the gap side-by-side to prevent shifting of the implanted bridge and ensure stable apposition with the rostral and caudal spinal cord over time. A length standard was used to create the hemisection gap, to ensure that the length of the bridge would be greater than the length of the hemisection and ensure good apposition of the multichannel bridge to the spared rostral and caudal spinal cord. PLG multichannel bridges were implanted into the gap immediately after SCI in Thy1-eGFP (JAX 007788; $N=3$ ). We repeated the experiment on three different animals, with the best case represented in the figures except for Fig. 3(d) where we show the variability of neuronal regrowth in the other two animals we tested. The laminectomy site was covered with gel foam to prevent the formation of muscle adhesions. Muscle was sutured over the laminectomized region with 5-0 chromic gut, and skin was closed with wound clips. Animals were maintained under bladder care for 8 weeks according to IACUC approved protocols. Postoperative care also included administration of Baytril (enrofloxacin $2.5 \mathrm{mg} / \mathrm{kg}$, once a day for 2 weeks), buprenorphine $(0.1 \mathrm{mg} / \mathrm{kg}$ twice a day for 3 days), and lactated ringers solution ( $5 \mathrm{~mL} / 100 \mathrm{~g}$, once a day for 5 days). Mice were euthanized 12 weeks postlesion/bridge implantation; mice were anesthetized with sodium pentobarbital $(100 \mathrm{mg} / \mathrm{kg}$, i.p.) and transcardially perfused with $30 \mathrm{ml}$ phosphate-buffered saline (PBS). Spinal cords were dissected based on vertebral level and the C2-T1 region collected for sectioning followed by THG imaging and histology.

\subsection{Microscopy and Live Imaging}

Live imaging was performed at day 0 , immediately before SCI and bridge implantation to establish a baseline, and 8 weeks post-SCI and PLG bridge implantation. Animals were anesthetized using isoflurane (2\%) and kept on a warming pad to maintain temperature at $37^{\circ} \mathrm{C}$ for all procedures; live imaging was completed within a 1-h time limit after the animals were anesthetized [Fig. 2(b)]. During imaging, the vertebral column was stabilized by clamping forceps placed on the C4 and C6 vertebrae and attached to a fixation apparatus to prevent movement of the cord. Images were acquired using an commercial upright microscope (Olympus BX module, Center Valley, Pennsylvania) coupled to a tunable femtosecond pulsed Ti:sapphire laser (Mai Tai HP with pulse-width compensator unit DeepSee, Spectra Physics, Santa Clara, California) working at $80 \mathrm{MHz}$. The setup was equipped with two digital photon counting PMTs (H7422P, Hammatsu, Japan) in the reflective path and a wide area PMT (R7600P-300, Hamamatsu, Japan) in the transmission path of excitation. A photon time tagging unit capable of lifetime imaging (FLIM-Box, ISS, UrbanaChampaign, Illinois) was used to collect the signals from the PMTs, enabling simultaneous detection and imaging. The imaging and data analysis were performed using the lab-made software (SimFCS, LFD). The third-harmonic images were imaged at $1038 \mathrm{~nm}$ (THG at $\sim 346 \mathrm{~nm}$ ) and collected with a narrow bandpass filter combination of glass filters (BG39 and UG11, Edmund Optics, Barrington, New Jersey) centered at $360 \pm 25 \mathrm{~nm}$. The single-photon confocal imaging was done using microscope's internal 473-nm diode laser. Images were collected by raster scanning at fixed pixel dwell time of $20 \mu \mathrm{s} /$ pixel and image size of $256 \times 256$. The scanning was controlled by SimFCS for THG and Olympus Fluoview for confocal imaging. A schematic representation of the microscope and data collection is shown in Fig. 1 .

\subsection{Third-Harmonic Generation Imaging}

Spinal cords were obtained from the same animals used for live imaging 12 weeks post-SCI and bridge implantation. The cords were cryosectioned horizontally at $160 \mu \mathrm{m}$ and sections 


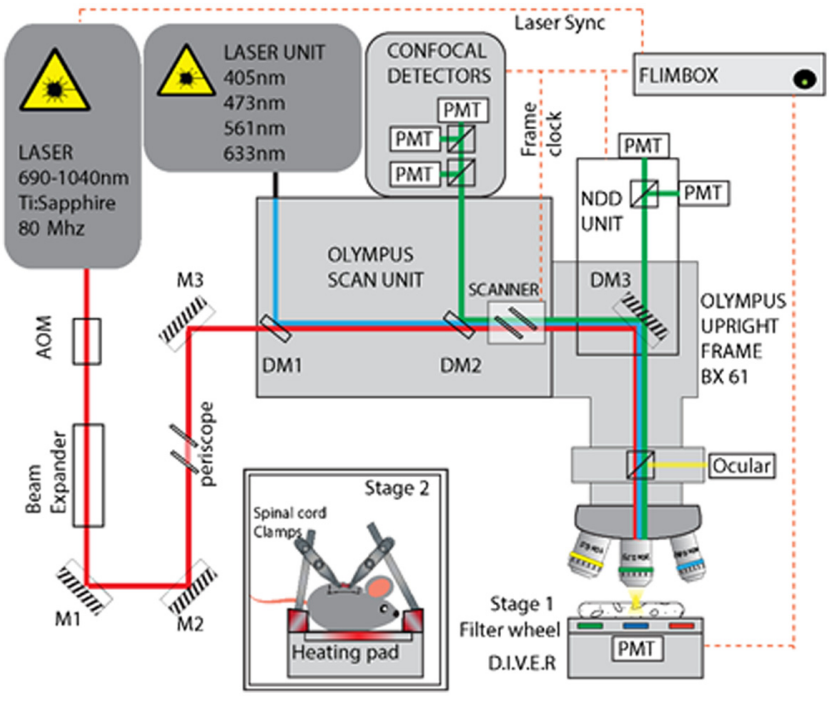

Fig. 1 Schematic representation for the microscope setup. The ultrafast laser system is coupled to the auxiliary input of the commercial upright laser scanning microscope system using an AOM (acoustic optical modulator) for power control and a beam expander to tune the beam size (telescope). The light is launched into an elevated scanning unit using a periscope-mirror set (M1, M2, and M3). The internal single-photon lasers are combined to the auxiliary laser at the DM1 dichroic mirror. The excitation laser goes through the XY galvano-scanner mirror unit and is launched through the microscope frame BX61 to the sample. The emission is collected using three separate geometries: (a) the nondescanned detection path (NDD), which is split from DM3 dichroic mirror, (b) the confocal unit with variable pinhole size from DM2 dichroic mirror, and (c) the transmission path detection using DIVER module, which uses combinational filters. The DIVER unit has an in-built filter wheel that can tune into visible and ultraviolet filters. Conventional samples can be mounted on stage 1 , the commercial stage, and a second animal mounting stage with heating pad can be mounted on top of DIVER module (stage 2). The red bold line shows the multiphoton, the blue line shows single-photon excitation, green line shows the emission path, yellow shows the ocular path, the dashed-orange line shows the signal lines, and the black line shows the fiber.

evaluated for THG imaging. We perfused the animals using cold PBS and we carefully removed the cord segment C2-T1. Cord were immediately frozen using Histobath (Thermo Shandon). Cords were sectioned using cryostat (Thermo Scientific). For sectioning, spinal cords were carefully aligned to show linear apposition between the spinal parenchyma and bridge and reveal the bridge channels through which axonal regeneration occurs. ${ }^{5}$ We imaged the regions across the bridge $500-\mu \mathrm{m}$ rostral and caudal, respectively. A large area detector for deep tissue imaging termed as the DIVER ${ }^{18}$ was employed for enhanced transmitted THG collection. We used $1038 \mathrm{~nm}$ as excitation and we collected the signal using a bandpass filter at $320 \mathrm{~nm}$. This module has automated filter wheel switching between conventional fluorescence emission spectral bands and narrower THG bands. THG is a nonlinear optical phenomenon that is produced by sufficiently high energy/photon density obtained through tightly focused light of ultrafast pulses of short duration of hundred femtoseconds. Nonlinear microscopy always found its home in neuronal imaging because of the inherent challenge in imaging deep through a scattering brain tissue. ${ }^{19}$ Nonlinear fluorescence excitation methods, such as two-photon fluorescence, ${ }^{20}$ three-photon fluorescence, ${ }^{21}$ four-photon fluorescence, ${ }^{22,23}$ have been reported in multiphoton imaging.
Similarly, nonlinear scattering phenomena, such as secondharmonic generation or THG, are used in the same imaging modality. Fluorescence is bound by the absorption cross section (two photons) for the fluorophores (eGFP) and their values can be found in the literature. ${ }^{24-26}$ In contrast to absorption, THG imaging is a coherent process produced by the broken axial focal symmetry because of nonlinear susceptibility changes in the focal plane. The localized THG signal provides high axial resolution and excellent contrast of changes in refractive index. Briefly, refreshing the third-order nonlinear processes, the induced polarization from the third-order susceptibility $\left[\chi^{(3)}\right]$ is $P_{i}^{3}=\epsilon_{0} \sum_{x y z} \chi_{i}^{(3)} E_{w}$, where $E$ is the incident electric field of frequency $(w)$ and $X^{(3)}$ is a fourth-rank tensor that multiplies $E(x), E(y), E(z)$, the electric field in three axes. It can be proven that the essential phase-matching condition for THG is refractive indices relation: $n(w)-n(3 w)=0$, which restricts the THG only special cases where the refractive index of fundamental $(w)$ matches the refractive index of the THG $(3 w)$. We performed THG measuring on mice spinal cord slides as described in Sec. 2.2.

\section{Results}

For the live imaging of the cord, animals were maintained under anesthesia for the duration of the experiment and positioned under the microscope as shown [Fig. 2(b)]. An initial (0 day) live imaging session on the Thy1-eGFP animals was performed immediately prior to SCI and bridge implantation to establish the capacity to detect in vivo neuronal fibers. Using a 488-nm (blue) laser, we imaged the eGFP neuronal fibers through cord up to $140-\mu \mathrm{m}$ deep inside the tissue [Fig. 2(d)]. We were able to (a)
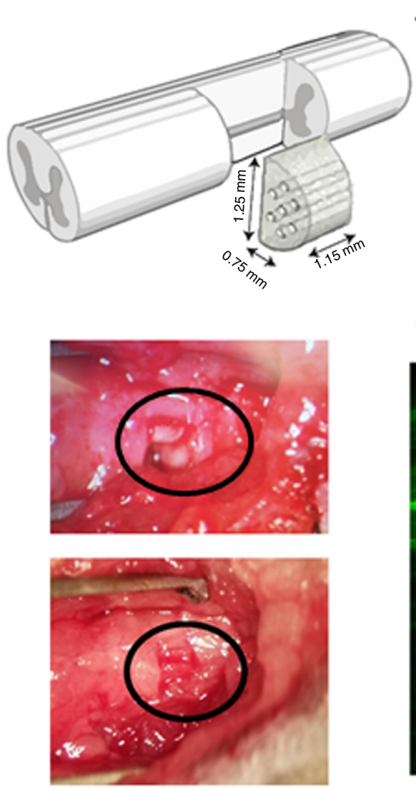

(c)
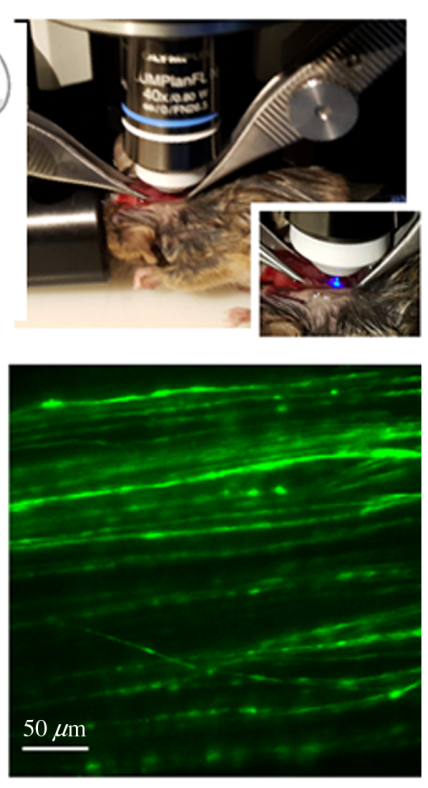

(d)
(140 $\mu \mathrm{m}$ MIP)

Fig. 2 (a) Schematic representation of PLG bridge implanted at C5 vertebral segment, (b) Thy-eGFP animal is positioned under microscope clamped on C4-C6 with exposed C5 segment, (c) picture showing $\mathrm{C} 5$ hemisection and bridges placed inside the black hole, and (d) circle imaging of the spinal cord prebridge implant showing neuronal fibers at the $\mathrm{C} 5$ level after bone removal. 
observe very distinct neuronal fibers inside the cord, expressing eGFP.

After the initial imaging session, we performed a C5 hemisection and bridge implantation as described in Sec. 2.2 [Figs. 2(a) and 2(c)]. For bridge implantation, the dimensions of the biomaterial bridge were adapted to the lesion size to ensure apposition within the lesion margins of the hemisection. A second live imaging session was performed 8 weeks post-SCI and bridge implantation, with anesthesia and acquisition time limited to $1 \mathrm{~h}$ for data collection. We used reflectance microscopy to detect the bridge and green fluorescence (eGFP) to image the neuronal fibers. We imaged rostral to and within the PLG bridge to establish feasibility to identify regrown fibers entering the bridge and traversing through it. Figure 3(a) shows a schematic representation of the bridge placed inside the spinal cord at C5 level. Numerous green fluorescent neuronal fibers were observed to accumulate in the rostral parenchyma [Fig. 3(b)], where they were positioned immediately adjacent to the entrance to the PLG bridge. Although few fibers were found to enter the bridge channels at this timepoint in the Thy 1 model, we were able to observe some [Figs. 3(c) and 3(d)]. These fibers reached distances of up to $\sim 500 \mu \mathrm{m}$ from the rostral side of the injury edge, progressing toward the caudal margin.

Following the imaging session, animals were resutured and maintained for an additional 4 weeks. At 12 weeks post-SCI and bridge implantation, animals were euthanized as described in Sec. 2 to confirm the observation of regenerated fibers within the bridge in histological sections and to test THG imaging for the visualization of myelinated fibers within the bridge. C2-T1 spinal cord segments were cryosectioned horizontally at $160 \mu \mathrm{m}$ to enable comparison of injured and uninjured spinal parenchyma, as well as regions rostral to, within, and caudal to the implanted bridge. Cryosections were mounted on glass slides as described in Sec. 2. (a)

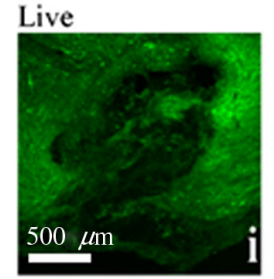

Cord- AF

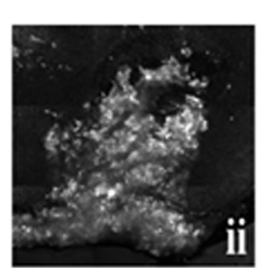

Bridge(reflectance)

(b) Sections
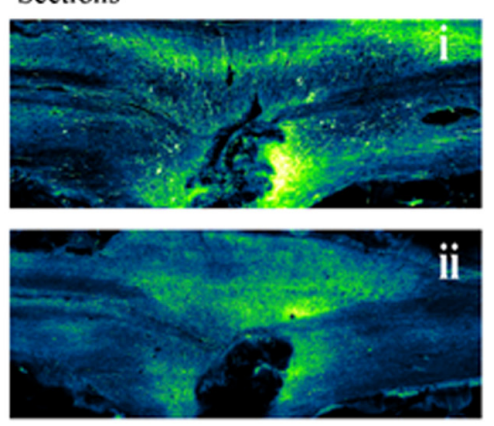

ii
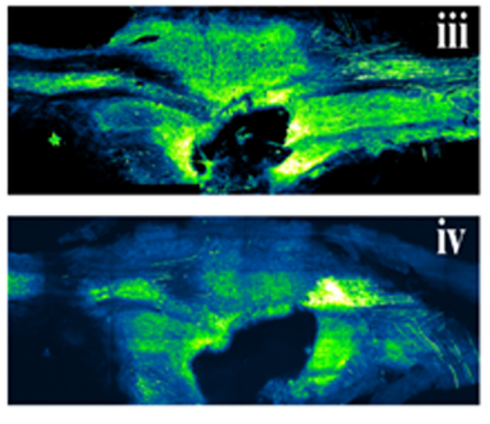

IV
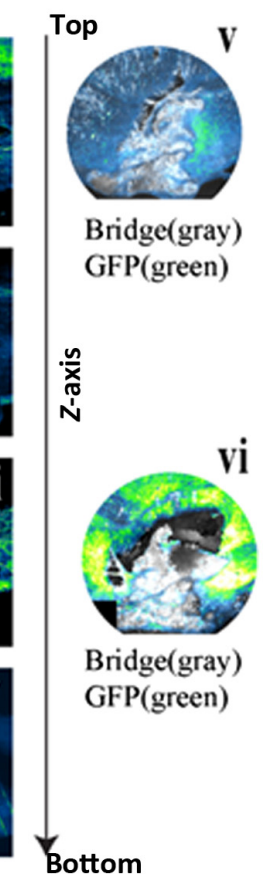

Bridge(gray) GFP(green)

告

Fig. 4 (a) Imaging of the bridge area showing the fibers (eGFP) are passing through it, in gray is the degrading bridge and (b) $Z$ stack imaging of the spinal cord after sectioning, in gray is the bridge and the fluorescent neuronal fibers are in green. (a)

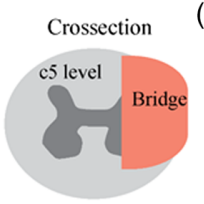

(c) Inside bridge

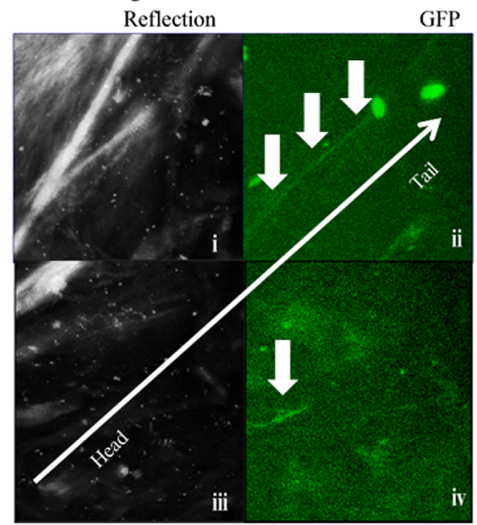

(b)

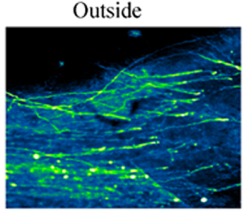

GFP (d)

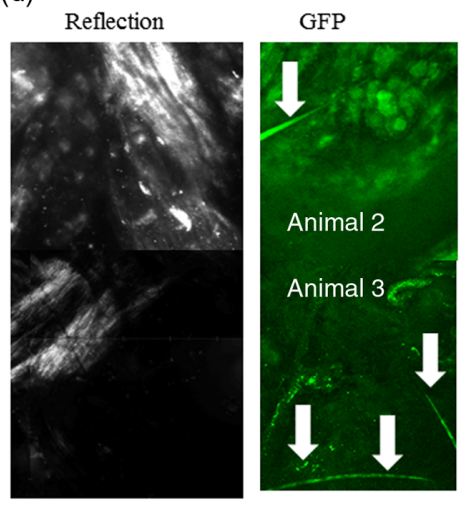

Fig. 3 (a) Schematic representation of a C5 cross section after bridge implant, (b) neuronal fibers (green) at the rostral edge of the PLG bridge entrance, (c) left panel: reflection microscopy showing the bridge surface on and right panel: the arrows show the neuronal fiber growing into the bridge, and (d) representative images of two more animals we tested with variable results. 
PLG biomaterial bridges exhibit a degradation profile. ${ }^{16}$ Using these procedures, we were able to observe the degrading bridges inside the tissue at C5 level [Fig. 4(a)] and histologically verify the presence of regenerating neuronal fibers. We observed increased green fluorescence (eGFP) arising from the area proximal to the bridges in both rostral and caudal area [Figs. 4(a) and 4(b)]. We speculate the presence of high fluorescence at bridge boundaries is due to the regenerating neuronal fibers accumulating at the bridge entrance. In Fig. 4(a), the green fluorescence is evidently present across the whole injury, suggesting that many more neuronal fibers have crossed the bridge in the 4 weeks after the live imaging session. This timecourse is in agreement with our previous publications in which we observed the neuronal fibers starting to enter the bridge and crossing through it in 4 to 12 weeks postinjury. ${ }^{5}$

Additionally, we tested the detection of THG signal within the bridge, as described in Sec. 2. THG has been demonstrated to be capable of imaging compact myelin in Schwann cells and in the CNS ex vivo. ${ }^{12,14}$ We tested this possibility by applying the same method to study the remyelination of the spinal cord after injury. Figure 5(a) illustrates detection of GFP+ axons in the rostral spinal parenchyma adjacent to the bridge using region reflectance microscopy. Figure 5(b) demonstrates successful detection of THG signal associated with these axons. However, while we were able to detect THG from neuronal fibers in the rostral parenchyma and fluorescent eGFP+ fibers throughout the bridge structure [Fig. 5(c)], we were unable to detect any THG signal within the bridge [Fig. 5(d)]. Taken together, these data suggest that the myelination is a process that does not directly follow the neuronal fibers regeneration and could occur at a later time.

\section{Discussion}

Evaluation of axonal damage, myelination, and axonal regeneration is critical to investigate the dynamics of regeneration after SCI. In vivo studies of neuronal fibers after SCI would improve knowledge of regenerating fibers growing into and through a permissive environment, such as the PLG bridge. This approach is also important as a strategy for improving understanding of axonal regeneration and sprouting in the spared parenchyma of the spinal cord after injury. Visualizing the regrowth of the neuronal fibers would enable us to investigate the direction of regeneration and eventually investigate any physical barrier slowing the regeneration process.

For many fiber tracts, reconstructing a circuit through regeneration or regenerative sprouting may be only the first step in establishing functional connectivity. In this regard, myelination of these new fibers will in many cases be a determining factor. Assessing the level of axonal myelination in vivo is a challenging task because the third-harmonic signal is easily absorbed from the tissues and along the microscope pathway, limiting the detection signal. However, it is possible to obtain (a)

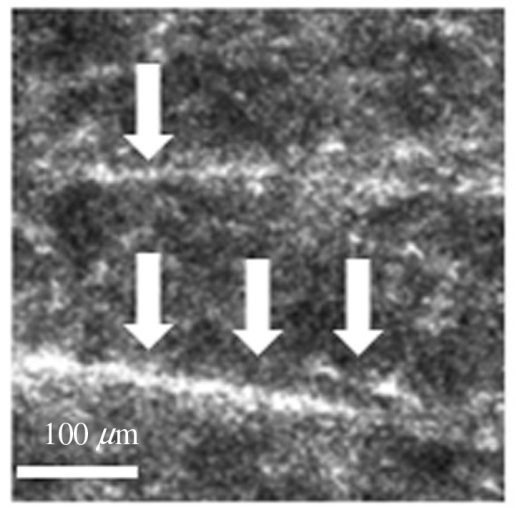

(c)

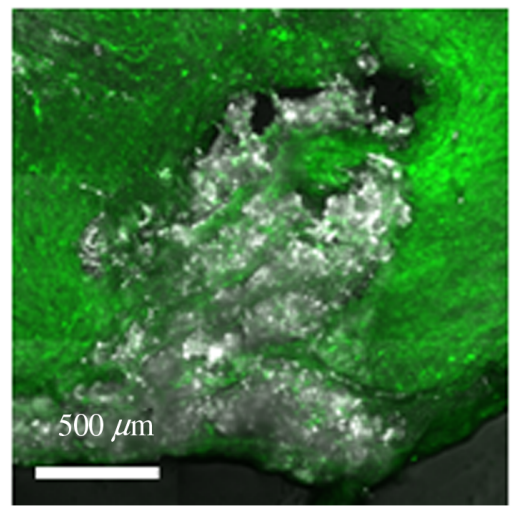

(b)

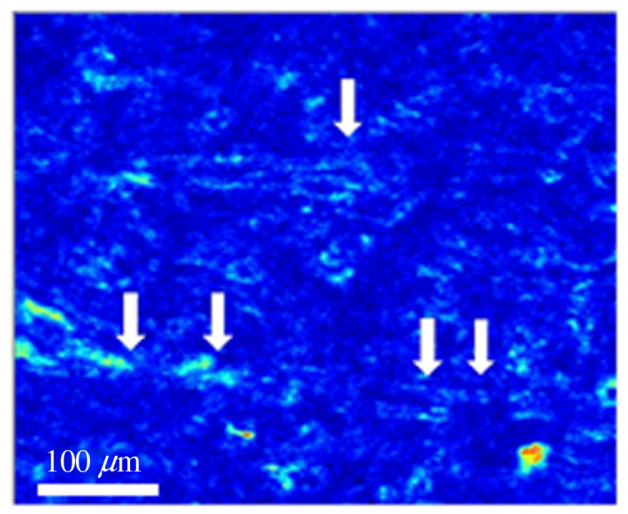

(d)

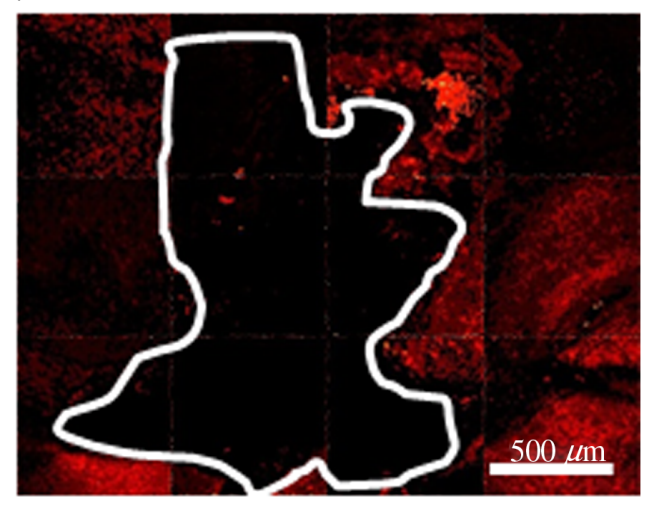

Fig. 5 (a) Reflecting microscopy showing axons on the rostral entrance of the bridge, (b) THG originating from myelin surrounding the neuronal fibers in panel (a), (c) imaging showing the neuronal fibers around and through the bridge and in gray is the bridge itself, and (d) THG is absent in the bridge area while signal is detected from the surrounding areas. 
myelination data from ex vivo fibers. Here, we demonstrate the feasibility of combining live imaging of neuronal regrowth at two separate time-points, before and after the PLG scaffold implantation, with ex vivo determination of myelination status of these fibers. Myelination in the spinal cord after PLG bridges implant has been demonstrated previously to be very low. ${ }^{27}$ When PLG bridges were implanted without trophic factors or sonic hedgehog only $18 \%$ of fibers exhibited colabeling for neurofilament and myelin basic protein 8 weeks postimplant. Although we were unable to detect any signal arising from THG in the PLG bridge area, we cannot exclude that the absence of myelination is due to the properties of the bridge. For example, the signal arising from the myelin could be quenched given the low proportion of myelinated fibers at this time point. Additionally, THG has only been used to visualize mature myelinated structures, in which compact myelin has formed; $;^{11,12}$ thus, it is also possible that we were unable to visualize THG signal due to the immature state of the myelin at this time point. Critically, three-photon imaging and THG microscopy have the potential to go deeper than two-photon imaging schemes, owing to greater penetration depth due to using a longer wavelength. Three-photon imaging and THG microscopy also render higher resolution images because of the spatial excitation photon density in a third-order nonlinear process. However, one main hurdle in increasing depth of imaging is the wavefront distortion suffered by the excitation beam through scattering in the layers of penetrated tissue thinning excitation photon density. This can be corrected by adaptive optics schemes ${ }^{28}$ and other photon density optimizing techniques to focus the light at one point using objective lens optimization. ${ }^{29}$ Future development of these systems will lead to an improvement into the live imaging of the spinal cord and to detect myelination in an in vivo setting.

\section{Disclosures}

No potential conflicts of interest relevant to this article were reported.

\section{Acknowledgments}

Funding for this work was provided by the National Institutes of Health (NIH) 5R01EB005678-12 (L.D.S., A.J.A., and B.J.C.) and the Christopher and Dana Reeve Foundation (A.J.A.) and NIH P41-RR03155 (M.A.D.).

\section{References}

1. A. E. Chiodo et al., "Spinal cord injury medicine. 5. Long-term medical issues and health maintenance," Arch. Phys. Med. Rehabil. 88, S76-S83 (2007).

2. J. Silver and J. H. Miller, "Regeneration beyond the glial scar," Nat. Rev. Neurosci. 5, 146-156 (2004).

3. J. L. Nielson, M. K. Strong, and O. Steward, "A reassessment of whether cortical motor neurons die following spinal cord injury," J. Comp. Neurol. 519, 2852-2869 (2011).

4. P. Freund et al., "Degeneration of the injured cervical cord is associated with remote changes in corticospinal tract integrity and upper limb impairment," PLoS One 7, e51729 (2012).

5. K. Pawar et al., "Biomaterial bridges enable regeneration and re-entry of corticospinal tract axons into the caudal spinal cord after SCI: association with recovery of forelimb function," Biomaterials $\mathbf{6 5}, 1-12$ (2015).
6. A. M. Thomas et al., "Channel density and porosity of degradable bridging scaffolds on axon growth after spinal injury," Biomaterials 34, 2213-2220 (2013).

7. A. Vats et al., "Scaffolds and biomaterials for tissue engineering: a review of clinical applications," Clin. Otolaryngol. Allied Sci. 28, 165-172 (2003).

8. M. J. Farrar et al., "Chronic in vivo imaging in the mouse spinal cord using an implanted chamber," Nat. Methods 9, 297-302 (2012).

9. G. Feng et al., "Imaging neuronal subsets in transgenic mice expressing multiple spectral variants of GFP," Neuron 28, 41-51 (2000).

10. C. Laperchia et al., "Two-photon microscopy imaging of thy1GFP-M transgenic mice: a novel animal model to investigate brain dendritic cell subsets in vivo," PLoS One 8, e56144 (2013).

11. A. J. Schain, R. A. Hill, and J. Grutzendler, "Label-free in vivo imaging of myelinated axons in health and disease with spectral confocal reflectance microscopy," Nat. Med. 20, 443-449 (2014).

12. M. J. Farrar et al., "In vivo imaging of myelin in the vertebrate central nervous system using third harmonic generation microscopy," Biophys. J. 100, 1362-1371 (2011).

13. E. M. Gibson, A. C. Geraghty, and M. Monje, "Bad wrap: myelin and myelin plasticity in health and disease," Dev. Neurobiol. 78, 123-135 (2018).

14. H. Lim et al., "Label-free imaging of Schwann cell myelination by third harmonic generation microscopy," Proc. Natl. Acad. Sci. U. S. A. 111, 18025-18030 (2014).

15. H. M. Tuinstra et al., "Multifunctional, multichannel bridges that deliver neurotrophin encoding lentivirus for regeneration following spinal cord injury," Biomaterials 33, 1618-1626 (2012).

16. L. De Laporte et al., "Plasmid releasing multiple channel bridges for transgene expression after spinal cord injury," Mol. Ther. 17, 318326 (2009).

17. M. J. Moore et al., "Multiple-channel scaffolds to promote spinal cord axon regeneration," Biomaterials 27, 419-429 (2006).

18. V. Crosignani, A. S. Dvornikov, and E. Gratton, "Enhancement of imaging depth in turbid media using a wide area detector,' J. Biophotonics 4, 592-599 (2011).

19. W. R. Zipfel, R. M. Williams, and W. W. Webb, "Nonlinear magic: multiphoton microscopy in the biosciences," Nat. Biotechnol. 21, 13691377 (2003).

20. W. Denk, J. H. Strickler, and W. W. Webb, "Two-photon laser scanning fluorescence microscopy," Science 248, 73-76 (1990).

21. S. Maiti et al., "Measuring serotonin distribution in live cells with threephoton excitation," Science 275, 530-532 (1997).

22. S. W. Chu et al., "Simultaneous four-photon luminescence, third-harmonic generation, and second-harmonic generation microscopy of GaN," Opt. Lett. 30, 2463-2465 (2005).

23. F. E. Hernandez et al., "Three- and four-photon absorption of a multiphoton absorbing fluorescent probe," Appl. Opt. 43, 5394-5398 (2004).

24. M. A. Albota, C. Xu, and W. W. Webb, "Two-photon fluorescence excitation cross sections of biomolecular probes from 690 to $960 \mathrm{~nm}$," Appl. Opt. 37, 7352-7356 (1998).

25. L. C. Cheng et al., "Measurements of multiphoton action cross sections for multiphoton microscopy," Biomed. Opt. Express 5, 3427-3433 (2014).

26. C. Xu et al., "Multiphoton fluorescence excitation: new spectral windows for biological nonlinear microscopy," Proc. Natl. Acad. Sci. U. S. A. 93, 10763-10768 (1996).

27. A. M. Thomas et al., "Sonic hedgehog and neurotrophin-3 increase oligodendrocyte numbers and myelination after spinal cord injury," Integr. Biol. 6, 694-705 (2014).

28. G. Norris et al., "A promising new wavelength region for three-photon fluorescence microscopy of live cells," J. Microsc. 246, 266-273 (2012).

29. D. Sinefeld et al., "Adaptive optics in multiphoton microscopy: comparison of two, three and four photon fluorescence," Opt. Express 23, 31472-31483 (2015).

Biographies for the authors are not available. 